\title{
Data Article
}

\section{Data supporting the assessment of biomass based electricity and reduced GHG emissions in Cuba}

\author{
Alexis Sagastume Gutiérrez ${ }^{\mathrm{a}, *}$, Juan J. Cabello Eras ${ }^{\mathrm{a}, \mathrm{b}}$, \\ Carlo Vandecasteele ${ }^{\mathrm{c}}$, Luc Hens ${ }^{\mathrm{d}}$ \\ ${ }^{a}$ Universidad de la Costa, Calle 50 No 55-66, PBX 3362200 Barranquilla, Colombia \\ ${ }^{\mathrm{b}}$ Universidad de Cienfuegos, Carretera a Rodas kilómetro 4, Cuatro caminos, Cienfuegos, Cuba \\ ${ }^{\mathrm{C}}$ Department of Chemical Engineering, KU Leuven, Celestijnenlaan 200F, 3001 Leuven, Belgium \\ d Vlaamse Instelling voor Technologisch Onderzoek (VITO), Boeretang 200, B2400 Mol, Belgium
}

\section{A R T I C L E I N F O}

\section{Article history:}

Received 27 November 2017

Received in revised form

11 January 2018

Accepted 25 January 2018

Available online 1 February 2018

\begin{abstract}
A B S T R A C T
Assessing the biomass based electricity potential of developing nations like Cuba can help to reduce the fossil fuels dependency and the greenhouse gas emissions. The data included in this study present the evolution of electricity production and greenhouse gas emissions in Cuba. Additionally, the potentialities to produce biomass based electricity by using the most significant biomass sources in Cuba are estimated. Furthermore, estimations of the potential reductions of greenhouse gas emissions, resulting from implementing the biomass based electricity potential of the different sources discussed in the study, are included. Results point to the most promising biomass sources for electricity generation and their potential to reduce GHG emissions.
\end{abstract}

(c) 2018 The Authors. Published by Elsevier Inc. This is an open access article under the CC BY license (http://creativecommons.org/licenses/by/4.0/).

\footnotetext{
DOI of original article: https://doi.org/10.1016/j.jclepro.2017.11.209

* Corresponding author.

E-mail address: asagastu1@cuc.edu.co (A. Sagastume Gutiérrez).
} 


\section{Specifications table}

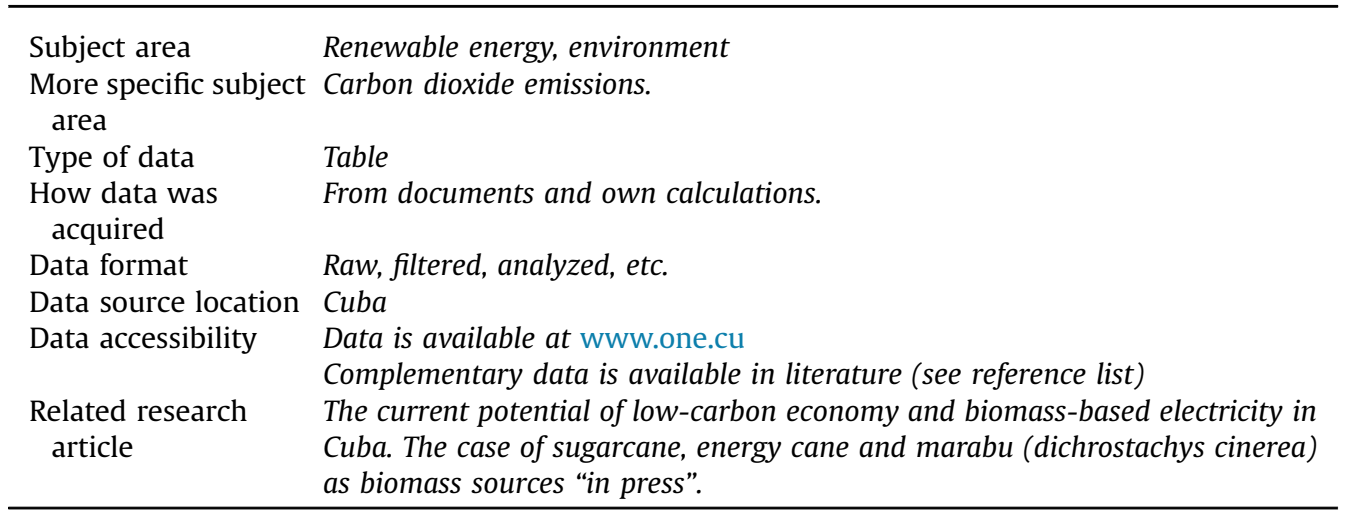

\section{Value of the data}

- This data contains key information for the biomass production and the GHG emissions in Cuba.

- This data can be used to estimate the biomass based electricity potential of Cuba.

- This data can be used to estimate the reduction of GHG emissions that could result from implementing the different biomass based electricity potentialities existing in Cuba.

- This data permits to focus on the largest biomass sources for energy production in Cuba.

\section{Data}

The data presented in the article is related to the research article: The current potential of lowcarbon economy and biomass-based electricity in Cuba. The case of sugarcane, energy cane and marabu (dichrostachys cinerea) as biomass sources [1]. The data corresponds to the evolution of the electricity production and of the GHG emissions in Cuba, and includes the biomass potential of the largest sources and the estimation of the associated biomass based electricity generation and greenhouse gas (GHG) emissions potential. The data of the evolution of the electricity production and of the GHG emissions was collected from the National Statistics Office of Cuba, when needed complemented with information from literature and databases. The estimations of the potentialities of biomass based electricity production and GHG emissions reduction in Cuba are calculated to highlight the main features.

\section{Materials and methods}

Based on the available biomass sources (between 2011 and 2016) estimations of the biomass based electricity potential and the possibilities to reduce GHG are developed. The biomass based electricity potential was calculated as:

$$
E=L H V_{\mathrm{w}} \cdot \eta_{\text {elect }}
$$

where:

$$
\text { E - Electricity potential }(\mathrm{kWh} / \mathrm{t})
$$


Table 1

Biomass production factors.

\begin{tabular}{lllllll}
\hline Biomass & Sugarcane $(\mathbf{t})$ & Paddy rice $(\mathbf{t})$ & Poultry (head) & Pig (head) & Pig manure (t) & Ref. \\
\hline Filter cake $(\mathrm{kg})$ & 33 & - & - & - & - & \\
Rice husk $(\mathrm{t})$ & - & 0.22 & - & - & - & {$[5]$} \\
Drying wastes $(\mathrm{t})$ & - & 0.04 & - & - & - & {$[6]$} \\
Poultry manure $\mathrm{kg})$ & - & - & 0.12 & - & - & {$[6]$} \\
Pig manure $(\mathrm{kg})$ & - & - & - & - & 194.7 & {$[7]$} \\
Biogas $\left(\mathrm{m}^{3}\right)$ & - & - & - & - & & {$[8]$} \\
\hline
\end{tabular}

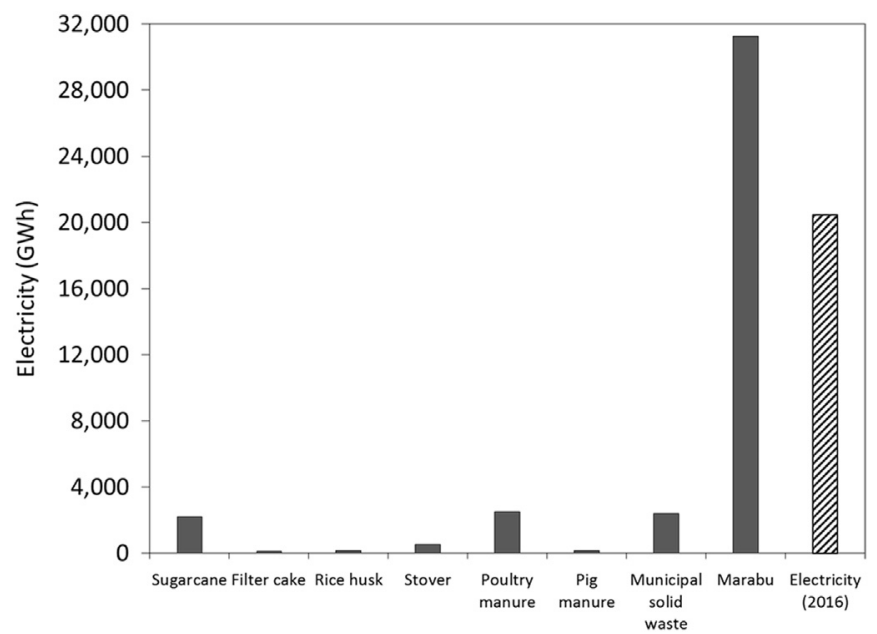

Fig. 1. Biomass based electricity potential of the biomass sources vs electricity generation in Cuba (2016).

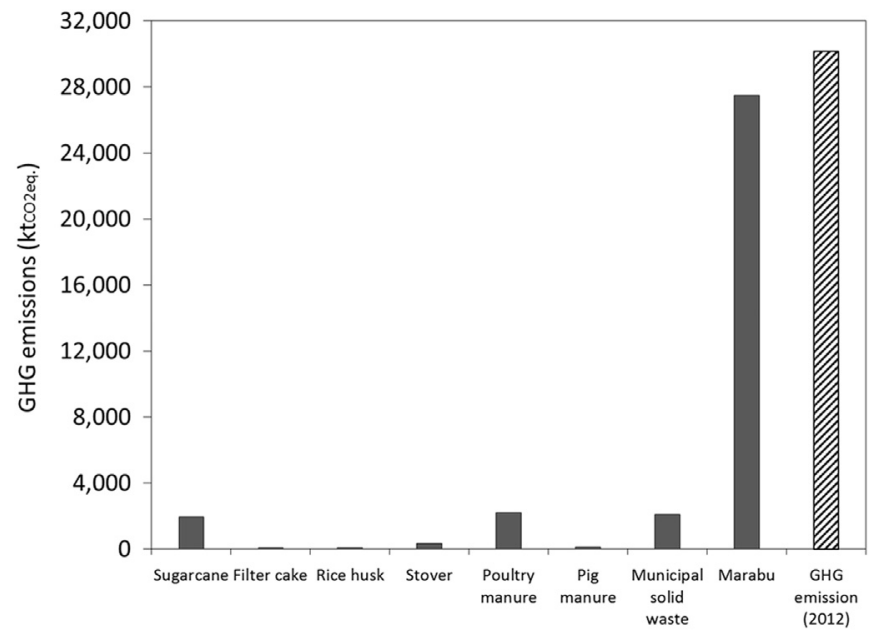

Fig. 2. Potential of GHG emission reductions of the biomass sources vs GHG emissions in Cuba (2012). 
Table 2

Evolution of power generation and GHG emissions in Cuba. (Source: [10-12]).

\begin{tabular}{|c|c|c|c|c|c|c|c|c|c|c|c|c|c|c|c|}
\hline Year & $\begin{array}{l}\text { Power } \\
\text { (MW) }\end{array}$ & $\begin{array}{l}\text { Electricity } \\
\text { (GWh) }\end{array}$ & $\begin{array}{l}\text { FBE } \\
\text { (GWh) }\end{array}$ & $\begin{array}{l}\text { SBE } \\
\text { (GWh) }\end{array}$ & $\begin{array}{l}\text { HP } \\
\text { (GWh) }\end{array}$ & $\begin{array}{l}\text { GHG.W } \\
\left(\mathbf{k t}_{\text {co2eq. }}\right)\end{array}$ & Year & $\begin{array}{l}\text { Power } \\
\text { (MW) }\end{array}$ & $\begin{array}{l}\text { Electricity } \\
\text { (GWh) }\end{array}$ & $\begin{array}{l}\text { FBE } \\
\text { (GWh) }\end{array}$ & $\begin{array}{l}\text { SBE } \\
\text { (GWh) }\end{array}$ & $\begin{array}{l}\text { HP } \\
\text { (GWh) }\end{array}$ & $\begin{array}{l}\text { ESPE } \\
\text { (GWh) }\end{array}$ & $\begin{array}{l}\text { GHG.C } \\
\left(\mathbf{k t}_{\text {co2eq. }}\right)\end{array}$ & $\begin{array}{l}\text { GHG.W } \\
\left(\mathbf{k t}_{\text {(OO2eq. }}\right)\end{array}$ \\
\hline 1959 & 475.6 & 2348.4 & 1956.4 & 392.0 & 5.9 & - & 1988 & 3841.3 & $14,542.3$ & $13,225.0$ & 1317.3 & 72.8 & 0.0 & - & 35,636 \\
\hline 1960 & 472.6 & 2492.7 & 2105.7 & 387.0 & 13.0 & 13,700 & 1989 & 3998.9 & $15,239.8$ & $13,959.7$ & 1280.1 & 82.0 & 0.0 & - & 35,739 \\
\hline 1961 & 509.1 & 2521.9 & 2086.9 & 435.0 & 8.5 & 12,182 & 1990 & 4077.9 & $15,024.7$ & $13,575.6$ & 1449.1 & 90.9 & 0.0 & 15,025 & 33,344 \\
\hline 1962 & 534.1 & 2552.5 & 2257.5 & 295.0 & 8.6 & 14,169 & 1991 & 4033.3 & $13,247.2$ & $11,982.8$ & 1264.4 & 104.7 & 0.0 & - & 29,710 \\
\hline 1963 & 532.1 & 2597.0 & 2345.0 & 252.0 & 49.8 & 13,040 & 1992 & 4032.2 & $11,538.0$ & $10,200.8$ & 1337.2 & 80.5 & 0.0 & 22,934 & 31,294 \\
\hline 1964 & 566.1 & 2811.4 & 2494.6 & 316.8 & 100.5 & 14,294 & 1993 & 4031.7 & $11,004.2$ & $10,117.0$ & 887.2 & 82.4 & 0.0 & - & 29,380 \\
\hline 1965 & 564.1 & 2954.5 & 2954.5 & - & 56.7 & 14,609 & 1994 & 4059.6 & $11,964.0$ & $11,067.1$ & 896.9 & 48.5 & 0.0 & 23,192 & 32,248 \\
\hline 1966 & 658.6 & 3157.4 & 3157.4 & - & 131.4 & 15,185 & 1995 & 3991.1 & $12,459.0$ & $11,769.3$ & 689.7 & 74.4 & 0.0 & - & 25,709 \\
\hline 1967 & 758.6 & 3453.6 & 3453.6 & - & 109.2 & 15,750 & 1996 & 4311.9 & $13,236.5$ & $12,314.4$ & 922.1 & 95.2 & 0.0 & 27,284 & 26,996 \\
\hline 1968 & 861.5 & 3615.4 & 3615.4 & - & 80.7 & 16,036 & 1997 & 4223.9 & $14,145.6$ & $13,275.9$ & 869.7 & 130.0 & 0.0 & - & 24,650 \\
\hline 1969 & 913.5 & 3782.3 & 3782.3 & - & 102.9 & 17,261 & 1998 & 4348.3 & $14,148.6$ & $13,369.5$ & 779.1 & 96.7 & 0.0 & 28,886 & 24,499 \\
\hline 1970 & 908.0 & 4888.5 & 4008.0 & 880.5 & 90.7 & 18,672 & 1999 & 4284.3 & $14,492.2$ & $13,611.3$ & 880.9 & 103.3 & 0.0 & - & 25,332 \\
\hline 1971 & 985.0 & 5020.5 & 4203.5 & 817.0 & 110.2 & 19,607 & 2000 & 4286.5 & $15,032.2$ & $14,088.0$ & 944.2 & 89.0 & 0.0 & 27,558 & 26,083 \\
\hline 1972 & 1466.2 & 5269.0 & 4624.0 & 645.0 & 74.0 & 20,799 & 2001 & 4410.9 & $15,299.8$ & $14,369.5$ & 930.3 & 75.0 & 0.0 & - & 25,453 \\
\hline 1973 & 1531.8 & 5707.9 & 4989.0 & 718.9 & 62.0 & 22,398 & 2002 & 3959.6 & $15,698.8$ & $14,760.3$ & 938.5 & 106.4 & 0.3 & 25,786 & 26,091 \\
\hline 1974 & 1644.6 & 6019.6 & 5283.4 & 736.2 & 89.4 & 22,911 & 2003 & 3965.0 & $15,810.5$ & $15,090.4$ & 720.1 & 127.7 & 0.4 & - & 25,486 \\
\hline 1975 & 1677.3 & 6588.9 & 5831.8 & 756.2 & 62.5 & 27,066 & 2004 & 3763.5 & $15,633.7$ & $14,845.1$ & 788.6 & 87.6 & 0.4 & 25,266 & 25,005 \\
\hline 1976 & 1704.6 & 7195.9 & 6422.6 & 773.3 & 53.2 & 27,224 & 2005 & 4275.1 & $15,341.1$ & $14,921.6$ & 419.5 & 67.7 & 0.1 & - & 26,006 \\
\hline 1977 & 1858.0 & 7705.0 & 6868.9 & 836.1 & 72.8 & 29,402 & 2006 & 5176.0 & $16,468.5$ & $16,062.4$ & 406.1 & 93.5 & 0.3 & 28,829 & 27,407 \\
\hline 1978 & 2288.3 & 8482.7 & 7527.0 & 955.7 & 83.2 & 30,689 & 2007 & 5429.4 & $17,622.5$ & $17,209.7$ & 412.8 & 121.4 & 0.2 & - & 26,795 \\
\hline 1979 & 2560.7 & 9403.1 & 8445.0 & 958.1 & 104.3 & 31,712 & 2008 & 5396.4 & $17,661.8$ & $17,127.6$ & 553.7 & 138.3 & 8.2 & 32,216 & 30,443 \\
\hline 1980 & 2731.4 & 9989.6 & 9035.4 & 954.2 & 97.1 & 31,401 & 2009 & 5550.0 & $17,727.1$ & $17,037.9$ & 534.8 & 150.8 & 3.6 & - & 29,897 \\
\hline 1981 & 2751.8 & $10,575.5$ & 9600.1 & 975.4 & 59.8 & 32,750 & 2010 & 5852.6 & $17,395.5$ & $16,832.3$ & 446.2 & 96.6 & 11.7 & 30,378 & 38,375 \\
\hline 1982 & 2974.5 & $11,071.4$ & $10,025.9$ & 1045.5 & 42.7 & 34,554 & 2011 & 5913.9 & $17,754.1$ & $17,186.6$ & 453.8 & 99.2 & 19.8 & - & 35,988 \\
\hline 1983 & 2999.9 & $11,551.4$ & $10,466.6$ & 1084.8 & 62.7 & 30,843 & 2012 & 5699.1 & $18,427.9$ & $17,744.3$ & 551.0 & 110.9 & 21.7 & 30,173 & 36,157 \\
\hline 1984 & 3111.2 & $12,292.0$ & $11,167.3$ & 1124.7 & 70.4 & 32,603 & 2013 & 6054.8 & $19,139.6$ & $18,306.9$ & 696.6 & 127.3 & 25.6 & - & 34,800 \\
\hline 1985 & 3249.0 & $12,199.4$ & $11,068.0$ & 1131.4 & 54.3 & 32,578 & 2014 & 6168.6 & $19,366.1$ & $18,588.3$ & 636.5 & 104.1 & 37.2 & - & 34,837 \\
\hline 1986 & 3419.2 & $13,176.4$ & $11,991.7$ & 1184.7 & 59.3 & 33,568 & 2015 & 6280.0 & $20,288.0$ & $19,585.3$ & 702.7 & 48.3 & 50.1 & - & - \\
\hline 1987 & 3532.0 & $13,594.0$ & $12,388.8$ & 1204.7 & 43.9 & 33,953 & 2016 & 6453.9 & $20,458.6$ & $19,648.0$ & 686.3 & 64.2 & - & - & - \\
\hline
\end{tabular}

${ }^{*}$ FBE - Fossil based electricity, SBE - Sugarcane based electricity, HE - Hydroelectricity, ESPE - Eolic + Solar photovoltaic, GHG.C - Net GHG emissions reported by the Cuban government, GHG.W - Net GHG emissions reported by the World Bank. 
Table 3

Evolution of sugarcane production and its use of agricultural land in Cuba. (Source: $[11,13]$ ).

\begin{tabular}{|c|c|c|c|c|c|c|c|c|c|}
\hline Year & $\begin{array}{l}\text { Harvested sur- } \\
\text { face (ha) }\end{array}$ & $\begin{array}{l}\text { Yield } \\
\text { (t) }\end{array}$ & $\begin{array}{l}\text { Production } \\
\text { (t) }\end{array}$ & $\begin{array}{l}\text { Bagasse } \\
(t)\end{array}$ & Year & $\begin{array}{l}\text { Harvested sur- } \\
\text { face (ha) }\end{array}$ & $\begin{array}{l}\text { Yield } \\
(\mathrm{t})\end{array}$ & $\begin{array}{l}\text { Production } \\
\text { (t) }\end{array}$ & $\begin{array}{l}\text { Bagasse } \\
(t)\end{array}$ \\
\hline 1959 & $1,070,000$ & 41.9 & $44,800,000$ & $12,960,000$ & 1988 & $1,297,300$ & 56.8 & $76,714,080$ & $21,819,600$ \\
\hline 1960 & $1,160,000$ & 40.9 & $47,500,000$ & $12,203,300$ & 1989 & $1,350,600$ & 60.0 & $85,218,000$ & $23,022,700$ \\
\hline 1961 & $1,260,000$ & 43.1 & $54,300,000$ & $14,002,700$ & 1990 & $1,420,300$ & 57.6 & $83,646,720$ & $23,261,900$ \\
\hline 1962 & $1,130,000$ & 32.5 & $36,700,000$ & $9,724,600$ & 1991 & $1,452,200$ & 54.9 & $79,698,330$ & $19,473,800$ \\
\hline 1963 & $1,070,000$ & 29.3 & $31,400,000$ & $8,386,100$ & 1992 & $1,451,700$ & 45.6 & $55,253,520$ & $10,093,300$ \\
\hline 1964 & $1,000,000$ & 37.2 & $37,200,000$ & $9,880,200$ & 1993 & $1,211,700$ & 36.0 & $44,960,400$ & $12,921,200$ \\
\hline 1965 & $1,060,000$ & 47.8 & $50,700,000$ & $13,344,100$ & 1994 & $1,248,900$ & 34.6 & $40,738,040$ & $12,902,700$ \\
\hline 1966 & 980,000 & 37.0 & $36,800,000$ & $9,874,900$ & 1995 & $1,177,400$ & 28.5 & $35,468,250$ & $10,208,100$ \\
\hline 1967 & $1,040,000$ & 35.0 & $50,500,000$ & $13,950,300$ & 1996 & $1,244,500$ & 33.2 & $41,377,160$ & $12,423,200$ \\
\hline 1968 & $1,010,000$ & 42.4 & $42,800,000$ & $11,869,000$ & 1997 & $1,246,300$ & 31.2 & $32,713,200$ & $11,859,500$ \\
\hline 1969 & 940,000 & 44.4 & $41,700,000$ & $11,551,400$ & 1998 & $1,048,500$ & 31.3 & $31,168,540$ & $10,070,300$ \\
\hline 1970 & $1,460,000$ & 55.8 & $81,500,000$ & $23,274,100$ & 1999 & 995,800 & 34.1 & $35,494,690$ & $10,673,300$ \\
\hline 1971 & $1,250,000$ & 41.7 & $52,200,000$ & $15,836,700$ & 2000 & $1,040,900$ & 35.6 & $35,852,760$ & $11,038,700$ \\
\hline 1972 & $1,180,000$ & 37.5 & $44,300,000$ & $13,369,100$ & 2001 & $1,007,100$ & 31.4 & $32,693,680$ & $11,599,000$ \\
\hline 1973 & $1,070,000$ & 45.0 & $48,200,000$ & $14,254,000$ & 2002 & $1,041,200$ & 33.3 & $21,438,540$ & $8,952,000$ \\
\hline 1974 & $1,100,000$ & 45.8 & $50,400,000$ & $14,779,200$ & 2003 & 643,800 & 34.3 & $22,672,300$ & $7,100,700$ \\
\hline 1975 & $1,180,000$ & 44.4 & $52,400,000$ & $15,153,300$ & 2004 & 661,000 & 36.0 & $18,619,200$ & $6,950,500$ \\
\hline 1976 & $1,220,000$ & 44.1 & $53,800,000$ & $15,275,800$ & 2005 & 517,200 & 22.4 & $8,895,040$ & $4,787,300$ \\
\hline 1977 & $1,140,000$ & 53.0 & $60,400,000$ & $16,073,200$ & 2006 & 397,100 & 28.0 & $9,226,000$ & $3,605,800$ \\
\hline 1978 & $1,240,000$ & 56.1 & $69,600,000$ & $18,678,800$ & 2007 & 329,500 & 36.1 & $13,728,830$ & $3,415,100$ \\
\hline 1979 & $1,310,000$ & 59.0 & $77,300,000$ & $19,585,100$ & 2008 & 380,300 & 41.3 & $17,953,110$ & $3,863,300$ \\
\hline 1980 & $1,390,000$ & 46.0 & $64,000,000$ & $17,108,000$ & 2009 & 434,700 & 34.3 & $14,797,020$ & $3,719,000$ \\
\hline 1981 & $1,210,000$ & 55.0 & $66,600,000$ & $19,147,000$ & 2010 & 431,400 & 26.7 & $13,512,870$ & $3,027,300$ \\
\hline 1982 & $1,330,000$ & 55.0 & $73,100,000$ & $19,075,000$ & 2011 & 506,100 & 31.2 & $11,272,560$ & $3,949,600$ \\
\hline 1983 & $1,200,000$ & 58.1 & $67,400,000$ & $19,149,000$ & 2012 & 361,300 & 39.9 & $15,971,970$ & $3,959,900$ \\
\hline 1984 & $1,350,000$ & 57.3 & $77,400,000$ & $19,635,000$ & 2013 & 400,300 & 40.3 & $16,329,560$ & $3,637,100$ \\
\hline 1985 & $1,347,800$ & 50.0 & $67,400,000$ & $18,315,000$ & 2014 & 405,200 & 44.10 & $19,300,000$ & $4,604,200$ \\
\hline 1986 & $1,328,600$ & 51.6 & $70,088,280$ & $19,584,000$ & 2015 & 435,600 & 44.30 & $19,297,080$ & $4,942,000$ \\
\hline 1987 & $1,358,300$ & 52.1 & $67,589,330$ & $19,969,000$ & 2016 & - & - & $15,806,667$ & $3,793,600$ \\
\hline
\end{tabular}

Table 4

Biomass properties and electric potential.

\begin{tabular}{llllll}
\hline Biomass & Moisture (\%) & $\left.\mathbf{H H V}_{\mathbf{d}} \mathbf{( M J} / \mathbf{k g}\right)$ & $\mathbf{L H V}_{\mathbf{w}}(\mathbf{M J} \mathbf{/ k g})$ & Electricity potential (kWh/t) $^{\text {Ref. }}$ \\
\hline Bagasse & $50.0 \%$ & 17.30 & 7.43 & 577.6 & {$[3]$} \\
Filter cake & $40.0 \%$ & 14.50 & 7.72 & 600.5 & {$[3]$} \\
Marabu & $19.0 \%$ & 20.70 & 16.30 & 1267.9 & {$[3]$} \\
Rice husk & $9.0 \%$ & 16.50 & 14.79 & 1150.7 & {$[14]$} \\
Maize & $6.1 \%$ & - & 15.68 & 1219.6 & {$[15]$} \\
Poultry manure & $39.7 \%$ & - & 8.54 & 664.2 & {$[15]$} \\
Pig manure & $92.1 \%$ & - & -1.24 & 0 & {$[15]$} \\
Biogas from pig manure & - & - & 18 & 51.7 & {$[9]$} \\
Municipal solid waste & $44.0 \%$ & - & 7.15 & 556 & {$[16]$} \\
\hline
\end{tabular}

$\eta_{\text {elect }}$ - Electricity efficiency of the generation technology (understand as the \% of the $L H V_{\mathrm{W} . \mathrm{B}}$. transformed into electricity)

An electricity production efficiency of $28 \%$ was considered for biomass incineration [1,3]. Moreover, to assess the potentialities of pig manure, where the use of the biogas resulting from manure rather than directly incinerating manure (because of its high moisture content) is considered, an electricity production efficiency of $35 \%$ was used [2]. 
Table 5

Production of the main crops, livestock and municipal solid wastes in Cuba: 2011-2016. (Source [11]).

\begin{tabular}{lllllll}
\hline Year & Sugarcane $(\mathbf{t})$ & Maize $(\mathbf{t})$ & Paddy rice $(\mathbf{t})$ & Poultry (heads) & Pig (heads) & Municipal solid waste $\left(\mathbf{m}^{\mathbf{3}}\right)$ \\
\hline $\mathbf{2 0 1 1}$ & $11,272,560$ & 304,800 & $3,256,100$ & $33,663,300$ & $3,256,100$ & $23,390,400$ \\
$\mathbf{2 0 1 2}$ & $15,971,970$ & 324,463 & $3,036,100$ & $30,182,000$ & $3,036,100$ & $27,817,400$ \\
$\mathbf{2 0 1 3}$ & $16,329,560$ & 354,000 & $3,366,700$ & $32,415,500$ & $3,366,700$ & $26,521,000$ \\
$\mathbf{2 0 1 4}$ & $19,300,000$ & 360,400 & $3,379,600$ & $32,285,800$ & $3,379,600$ & $27,221,300$ \\
$\mathbf{2 0 1 5}$ & $19,297,080$ & 426,200 & $3,492,800$ & $31,963,900$ & $3,492,800$ & $28,007,800$ \\
$\mathbf{2 0 1 6}$ & $15,806,667$ & 427,295 & $3,600,800$ & $31,336,200$ & $3,600,800$ & $28,796,400$ \\
\hline
\end{tabular}

Table 6

Estimation of the biomass production from the more significant sources in Cuba: 2011-2016.

\begin{tabular}{|c|c|c|c|c|c|c|c|c|}
\hline Year & $\begin{array}{l}\text { Bagasse } \\
(t)\end{array}$ & $\begin{array}{l}\text { Filter cake } \\
(t)\end{array}$ & $\begin{array}{l}\text { Rice husk } \\
(t)^{\mathrm{a}}\end{array}$ & $\begin{array}{l}\text { Stover } \\
(t)\end{array}$ & $\begin{array}{l}\text { Poultry man- } \\
\text { ure }(t)\end{array}$ & $\begin{array}{l}\text { Pig manure } \\
(t)\end{array}$ & $\begin{array}{l}\text { Municipal solid } \\
\text { waste (t) }{ }^{\mathrm{b}}\end{array}$ & Total $(t)$ \\
\hline 2011 & $3,949,600$ & 371,994 & 147,264 & 304,800 & $4,039,596$ & $2,587,623$ & $3,508,560$ & $14,909,437$ \\
\hline 2012 & $3,959,900$ & 527,075 & 166,816 & 324,463 & $3,621,840$ & $2,412,789$ & $4,172,610$ & $15,185,493$ \\
\hline 2013 & $3,637,100$ & 538,875 & 174,876 & 354,000 & $3,889,860$ & $2,675,516$ & $3,978,150$ & $15,248,378$ \\
\hline 2014 & $4,604,200$ & 636,900 & 152,048 & 360,400 & $3,874,296$ & $2,685,768$ & $4,083,195$ & $16,396,807$ \\
\hline 2015 & $4,942,000$ & 636,804 & 108,690 & 426,200 & $3,835,668$ & $2,775,728$ & $4,201,170$ & $16,926,259$ \\
\hline 2016 & $3,793,600$ & 521,620 & 133,652 & 427,295 & $3,760,344$ & $2,861,556$ & $4,319,460$ & $15,817,526$ \\
\hline
\end{tabular}

${ }^{\mathrm{a}}$ Includes rice husk and drying wastes.

${ }^{\mathrm{b}}$ A density of $150 \mathrm{~kg} / \mathrm{m}^{3}$ is considered for Municipal Solid Waste.

Table 7

Estimation of the marabu (dichrostachys cinerea) biomass stock in Cuba: 2011-2016.

\begin{tabular}{lll}
\hline Year & Surface (ha) & Biomass (t) \\
\hline $\mathbf{2 0 1 1}$ & $1,500,000$ & $55,500,000$ \\
$\mathbf{2 0 1 2}$ & $1,600,000$ & $59,200,000$ \\
$\mathbf{2 0 1 3}$ & $1,700,000$ & $62,900,000$ \\
$\mathbf{2 0 1 4}$ & $1,800,000$ & $66,600,000$ \\
$\mathbf{2 0 1 5}$ & $1,900,000$ & $70,300,000$ \\
$\mathbf{2 0 1 6}$ & $2,000,000$ & $74,000,000$ \\
\hline
\end{tabular}

Table 8

Calculation of biomass based electricity potential in Cuba: 2011-2016.

\begin{tabular}{lllllllll}
\hline Year & $\begin{array}{l}\text { Sugarcane } \\
\text { (GWh) }\end{array}$ & $\begin{array}{l}\text { Rice husk } \\
\text { (GWh) }\end{array}$ & $\begin{array}{l}\text { Stover } \\
\text { (GWh) }\end{array}$ & $\begin{array}{l}\text { Poultry man- } \\
\text { ure (GWh) }\end{array}$ & $\begin{array}{l}\text { Pig manure } \\
\text { (GWh) }\end{array}$ & $\begin{array}{l}\text { Municipal solid } \\
\text { waste (GWh) }\end{array}$ & $\begin{array}{l}\text { Marabu } \\
\text { (GWh) }\end{array}$ & $\begin{array}{l}\text { Total } \\
\text { (GWh) }\end{array}$ \\
\hline $\mathbf{2 0 1 1}$ & 1578 & 169 & 372 & 2683 & 134 & 1951 & 70,371 & 77,336 \\
$\mathbf{2 0 1 2}$ & 2236 & 192 & 396 & 2406 & 125 & 2320 & 75,062 & 82,848 \\
$\mathbf{2 0 1 3}$ & 2286 & 201 & 432 & 2584 & 138 & 2212 & 79,753 & 87,720 \\
$\mathbf{2 0 1 4}$ & 2702 & 175 & 440 & 2573 & 139 & 2271 & 84,445 & 92,878 \\
$\mathbf{2 0 1 5}$ & 2702 & 125 & 520 & 2548 & 144 & 2336 & 89,136 & 97,644 \\
$\mathbf{2 0 1 6}$ & 2213 & 154 & 521 & 2498 & 148 & 2402 & 93,827 & 101,873 \\
\hline
\end{tabular}

The factors used to estimate the biomass resulting from the production of different crops and livestock in Cuba are included in Table 1. For the estimations of dichrostachys cinerea (kwon as marabu, is a non-indigenous bush tree that is widely available and considered a fast spreading plague, 
Table 9

Calculation of the biomass based GHG reduction potential in Cuba: 2011-2016.

\begin{tabular}{|c|c|c|c|c|c|c|c|c|}
\hline Year & $\begin{array}{l}\text { Sugarcane } \\
\left(\mathbf{k t}_{\text {co2.eq }}\right)\end{array}$ & $\begin{array}{l}\text { Rice husk } \\
\left(\text { kt }_{\text {co2.eq }}\right)\end{array}$ & $\begin{array}{l}\text { Stover } \\
\left(\mathbf{k t}_{\text {co2.eq }}\right)\end{array}$ & $\begin{array}{l}\text { Poultry man- } \\
\text { ure (kt } \text { co2.eq })\end{array}$ & $\begin{array}{l}\text { Pig manure } \\
\left(\mathbf{k t}_{\text {co2.eq }}\right)\end{array}$ & $\begin{array}{l}\text { Municipal solid } \\
\text { waste }\left(\mathbf{k t}_{\mathbf{c o 2 . e q}}\right)\end{array}$ & $\begin{array}{l}\text { Marabu } \\
\left(\mathbf{k t}_{\text {co2.eq }}\right)\end{array}$ & $\begin{array}{l}\text { Total } \\
\left(\mathbf{k t}_{\text {co2.eq }}\right)\end{array}$ \\
\hline 2011 & 1387 & 112 & 245 & 2359 & 118 & 1715 & 61,856 & 67,860 \\
\hline 2012 & 1966 & 126 & 261 & 2115 & 110 & 2040 & 65,979 & 72,694 \\
\hline 2013 & 2010 & 133 & 285 & 2271 & 122 & 1945 & 70,103 & 76,967 \\
\hline 2014 & 2375 & 115 & 290 & 2262 & 122 & 1996 & 74,227 & 81,505 \\
\hline 2015 & 2375 & 82 & 343 & 2239 & 126 & 2054 & 78,351 & 85,687 \\
\hline 2016 & 1945 & 101 & 343 & 2195 & 130 & 2111 & 82,474 & 89,398 \\
\hline
\end{tabular}

occupying between 1.5 and 2 million ha) it is considered that between 2011 and 2016 its area increased from 1.5 to 2 million ha (at a rate of 100,000 ha/year). Marabu yields $37 \mathrm{t} /$ ha with a re-grow period of three years [3]. Based on re-grow period, the yearly marabu based electricity potential is estimated as $33.3 \%$ of the overall potential of the marabu stock.

To assess the potential reductions of the GHG emissions, it is considered that the GHG emissions of producing the different crops and livestock are allocated to the production of the product (e.g. rice, maize grain, meat, eggs, sugar, etc.). This is not entirely true since biomass is not carbon neutral. However, it serves as a first approximation. Thus, it is considered that biomass based electricity can save $100 \%$ of the GHG emissions resulting from generating the same amount of fossil based electricity. In Cuba, the greenhouse gas emission factor for electricity generation is $0.879 \mathrm{t}_{\mathrm{CO} 2} \mathrm{eq} . / \mathrm{MWh}$ [4] (Figs. 1 and 2, Tables 2-9).

\section{Transparency document. Supplementary material}

Supplementary data associated with this article can be found in the online version at http://dx.doi. org/10.1016/j.dib.2018.01.071.

\section{References}

[1] A. Sagastume, J.J. Cabello, D. Huisingh, C. Vandecasteele, L. Hens, The current potential of low-carbon economy and biomass-based electricity in Cuba. The case of sugarcane, energy cane and marabu (Dichrostachys cinerea) as biomass sources, J. Clean. Prod. 172 (2018) 2108-2122.

[2] P. Kaparaju, J. Rintala, Mitigation of greenhouse gas emissions by adopting anaerobic digestion technology on dairy, sow and pig farms in Finland, Renew. Energy 36 (2011) 31-41.

[3] A. Sagastume, J.J. Cabello, L. Hens, C. Vandecasteele, The biomass-based electricity generation potential of the province of Cienfuegos, Cuba, Waste Biomass Valoriz. 8 (2016) 2075-2085.

[4] J.J. Cabello, D. Garcia, A. Sagastume, R. Priego, L. Hens, C. Vandecasteele, An approach to sustainable development: the case of Cuba, Environ. Dev. Sustain. 14 (2012) 573-591.

[5] P.A. Ochoa, J.J. Cabello, A. Sagastume, L. Hens, C. Vandecasteele, Residue from sugarcane juice filtration (filter cake): energy use at the sugar factory, Waste Biomass Valoriz. 1 (2010) 407-413.

[6] L.M. Contreras, Digestión anaerobia de residuos de la agroindustria arrocera cubana para la producción de biogás (Ph.D. Thesis), Universidad Central "Marta Abreu" de Las Villas, Cuba, 2013.

[7] C.M. Williams, Poultry waste management in developing countries, Food Agric. Organ. U. N. Poult. Dev. Rev. 46 (2013) 1-2.

[8] A. Sagastume, J.J. Cabello, P. Billen, C. Vandecasteele, Environmental assessment of pig production in Cienfuegos, Cuba: alternatives for manure management, J. Clean. Prod. 112 (2016) 2518-2528.

[9] J.W. De Vries, T.M.W.J. Vinken, L. Hamelin, I.J.M. De Boer, Comparing environmental consequences of anaerobic mono-and co-digestion of pig manure to produce bio-energy - a life cycle perspective, Bioresour. Technol. 125 (2012) 239-248.

[10] ONE, Anuario estadístico de cuba 2016, Oficina Nacional de Estadística, La Habana, Cuba, 2008 (Available in: 〈http://www. one.cu/publicaciones/50aniversario/Estad\%C3\%ADsticas\%20Energ\%C3\%A9ticas\%20en\%20la\%20Revoluci\%C3\%B3n.pdf), Accessed 17 November 2017).

[11] ONE, Estadísticas energéticas de la revolución, Oficina Nacional de Estadística, La Habana, Cuba, 2017 (Available in: 〈http:// www.one.cu/aec2016.htm〉, Accessed 17 November 2017).

[12] World Bank, 2017. Available in: 〈https://data.worldbank.org/indicator/EN.ATM.CO2E.KT?Locations=CU\&view=map 〉, (Accessed 17 November 2017).

[13] O.A. Echevarría, Cuba and the international sugar market, Cuba Transit. 5 (1995) 363-373. 
[14] J.A. Suarez, C.A. Luengo, F.F. Felfli, G. Bezzon, P.A. Beaton, Thermochemical properties of Cuban biomass, Energy Sources 22 (2000) 851-857.

[15] Phyllies2, 2017. Available in: 〈https://www.ecn.nl/phyllis2〉, (Accessed 17 November 2017).

[16] M.C. Lloréns, M.L. Torres, H. Alvarez, A.P. Arrechea, J.A. García, S.D. Aguirre, A. Fernández, Characterization of municipal solid waste from the main landfills of Havana city, Waste Manag. 28 (2008) 2013-2021. 